\title{
Study on the Electronic Structures of ZnS and Ag-doped ZnS from Density Functional Theory

\author{
Ailing $\mathrm{Wu}^{1, \mathrm{a}}$, Yang Qiao ${ }^{1, \mathrm{~b}}$, Fengzheng $\mathrm{Lv}^{2, \mathrm{c}}$
}

1(School of Space Science and Physics, Shandong University at Weihai, Weihai 264209, China)

\author{
2 (Graduate School at Shenzhen, Tsinghua University, Shenzhen 518055,China) \\ aailingwu@sdu.edu.cn, b23572380@qq.com, 'Ifzlpdfengzhen@126.com
}

Keywords: density functional theory, electronic structure, Ag-doped ZnS

Abstract: The band structures and electronic structures of pristine and Ag-doped zinc blende $\mathrm{ZnS}$ were calculated with the ab-initio ultra-soft pseudopotential plane wave approximation method based on density functional theory (DFT). The results show that Ag-doping narrows the band gap of $\mathrm{ZnS}$ and the acceptor impurity level is introduced by importing impurity Ag. It is pointed out that the acceptor level is hybridization due to the overlapping of the Ag-4d and S-3p. By analysis of Mulliken populations, $\mathrm{Zn}-\mathrm{S}$ bond has strong covalence in $\mathrm{ZnS}$ and the covalence of bond Ag-S is weakest after Ag-doped.

\section{Introduction}

Over the past decades, Zinc sulfide $(\mathrm{ZnS})$ with a direct band gap of $3.68 \mathrm{eV}$ for the zinc-blende( $\mathrm{ZB})$ structure at room temperature ${ }^{[1]}$ has been attracted more attentions because of its potential applications in nano-optoelectronic and environmental remedy industries such as light emission diodes (LED), nano-sensors, dye-sensitized solar cells and photocatalysts ${ }^{[2-7]}$. However, the wavelength range of which pure $\mathrm{ZnS}$ materials can stimulate is limited, and pure $\mathrm{ZnS}$ material has some flaws which hinder it from further applied research. Hence, an effort to either modulate the band structure of $\mathrm{ZnS}$ to meet the special functions is being pursued. By doping different kinds of elements, the semiconductor material band gap can be reduced and the electronic structure can be improved, which helps to enhance the response of the visible light and the photocatalytic activity ${ }^{[8]}$.

The study found that $\mathrm{ZnS}$ is a kind of preferred substrate material to be doped with transition metal elements or rare earth elements as active particles, which provides convenient conditions for the modification of $\mathrm{ZnS}$ properties by doping. In chemical reactions, d-state electrons of transition metal atoms are all involved in the formation of chemical bonds. That means the d-state electrons can show a variety of oxidation properties. Because of the bonding empty d-state of the transiting metal, many ligands are easy to form stable coordination compound. In this mechanism the transiting metal shows a higher catalytic activity, especially the transition metal atoms doped semiconductor materials which have been the subject of much public concern. $\mathrm{ZnS}$ doped with transition metals Ag were investigated in detail.

The electronic structure of semiconductor material determines its physical properties. Based on the first-principles methods of density functional theory (DFT), we conducted analog calculations on the electronic structures of $\mathrm{ZnS}$ and Ag-doped $\mathrm{ZnS}$. The results show that Ag-doping narrows the band gap of $\mathrm{ZnS}$ and the acceptor impurity level is introduced by importing impurity Ag.

\section{Theoretical method and computational details}

Sphalerite $\mathrm{ZnS}$ was adopted in the calculation. We constructed $2 \times 1 \times 1 \mathrm{ZnS}$ supercell model and doped ones (shown in the Fig.1). At a quarter of unit cell body diagonals were $S$ atoms, likewise, at the eight top corners and six center of face were $\mathrm{Zn}$ atoms, each unit cell contained $4 \mathrm{Zn}$ atoms and $4 \mathrm{~S}$ atoms. Doped Ag atoms substituted respectively $\mathrm{Zn}$ atoms at $(0.5,0.5,0.5)$ in the unit cell, as shown in Fig.1. The calculations were performed using CASTEP code ${ }^{[9]}$ based on first-principles DFT. The external 
potential of the valence electron in the system took ultrasoft pseudopotential, the exchange and correlation interactions were modeled using the generalized gradient approximation (GGA) with the Perdew-Burke-Ernzerhof (PBE) function ${ }^{[10]}$. The energy cutoff (Ecut) of planewave functions was set to $350.0 \mathrm{eV}$. All atomic positions in the $\mathrm{ZnS}$ supercell had been relaxed according to the total energy and force using the BFGS scheme ${ }^{[11]}$, based on the cell optimization criterion (RMS force of $0.01 \mathrm{eV} / \AA$, stress of $0.02 \mathrm{GPa}$, and displacement of $5.0 \times 10^{-4} \AA$ ). The calculation of total energy and electronic structures was followed by cell optimization with SCF tolerance of $5.0 \times 10^{-7} \mathrm{eV} / \mathrm{atom}$. The $\mathrm{k}$ mesh points of $3 \times 6 \times 6$ under the Monkhorst-Pack programme ${ }^{[12]}$ were used in the calculation for integrations over the Brillouin zones of $\mathrm{ZnS}$ and $\mathrm{Ag}$ doped- $\mathrm{ZnS}$.

Atoms of the same species were completely equivalent in the $\mathrm{ZnS}$ crystal lattice. While the same species atoms which located different place had different population after importing impurity. So we had to classify them. Fig.1 showed that the blue atom was Ag, the gray atoms were $\mathrm{Zn}$ and the yellow were S. S atoms were classified two sorts: I sort atoms were signed 1,4,6,7 in the picture and II sort atoms connected with $\mathrm{Ag}$ directly were signed 2、3、5、8. $\mathrm{Zn}$ atoms were sorted four: Atoms marked 1 was I sort, II sort were marked with 2, III sort were marked with 5, and the others (signed 3、4、6、 7 in the picture) were IV sort.
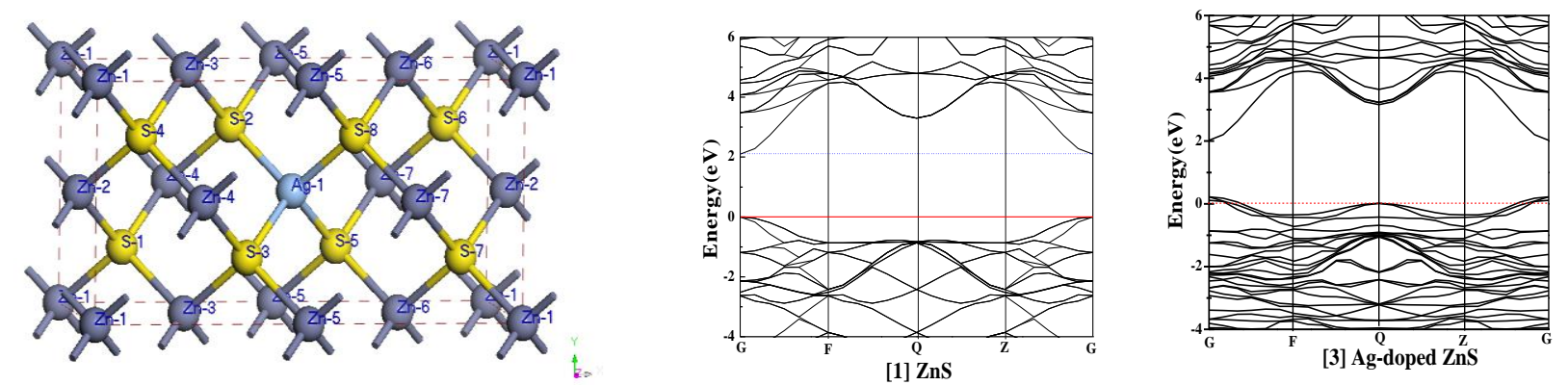

Fig.1. Structure for Ag-doped $\mathrm{ZnS}(2 \times 1 \times 1)$ supercell $\quad$ Fig. 2.The band structure of intrinsic system and doped $\mathrm{ZnS}$

\section{Results and Discussion}

Structural optimization: To assess the accuracy of our computation method, we performed a series of calculations for cell optimization. The equilibrium lattice constants are $a=b=c=0.54447 \mathrm{~nm}$ after optimization, which are in good agreement with experimental results $(a=b=c=0.54093 \mathrm{~nm}){ }^{[13]}$.

Electronic structure: Band structures of pristine $\mathrm{ZnS}$ and Ag-doped $\mathrm{ZnS}$ were presented in Fig.2. Zero of the band structure in Fig.2 was Fermi energy level. As illustrated in Fig.2-[1], the calculation shows a direct band gap of $2.097 \mathrm{eV}$ at the high symmetric G-point in the Brillouin zone for pristine $\mathrm{ZnS}$, which is lower than the experiment value of $3.68 \mathrm{eV}$. The bottom of conduction band and top of valence band both locate at $\mathrm{G}$ point, which is the representative characteristic for direct transition semiconductor. The band gap underestimation of DFT always exists as the result of the well-known limitation of predicting accurate energy band properties ${ }^{[14]}$. For Ag-doped ZnS, the calculated band gap value has a slight decrease compared with pristine $\mathrm{ZnS}$ (shown in Fig.2-[2]). Some levels pass through the Fermi level $\boldsymbol{E}_{\boldsymbol{f}}$, indicating that $\mathrm{Ag}$ doped in $\mathrm{ZnS}$ acts as the acceptor impurity. The narrow band gap and the acceptor states in the gap are beneficial for improving the visible-light absorption and photocatalysis.

The density of states (DOS) and partial density of states (PDOS) of ZnS and Ag-doped ZnS systems were shown in Fig.3. Mulliken population of pure $\mathrm{ZnS}$ and Ag-doped $\mathrm{ZnS}$ were shown in Table1. to Table 3.

As shown in Fig.3[1], conduction bands mainly dominated by the valence electrons of $\mathrm{Zn}(4 \mathrm{~s}, 4 \mathrm{p})$ and $\mathrm{S}(3 \mathrm{p})$ orbits in the pure $\mathrm{ZnS}$ system mainly locate in the 4.0 to $10.0 \mathrm{eV}$ energy range. A significant 
charge transfer from $\mathrm{Zn}(4 \mathrm{~s})$ to $\mathrm{S}(3 \mathrm{p})$ orbits shown in Table 1 and an overlap between the $\mathrm{Zn}(4 \mathrm{~s})$ and $\mathrm{S}(3 \mathrm{p})$ valence electrons depicted in Figure 3[1] reveal not only covalent bonding but also ionic bonding between the FNN(first nearest neighbor) $\mathrm{Zn}-\mathrm{S}$ in pure $\mathrm{ZnS}$. Valence bands in pure $\mathrm{ZnS}$ are mainly combined by two part, that, top valence bands ( 4.9 to $0 \mathrm{eV})$ and bottom valence bands (6.8 to $4.9 \mathrm{eV})$, as illustrated in Fig.3[1]. It is worth noting that the top valence bands and bottom valence bands are mainly contributed by $\mathrm{S}(3 \mathrm{p})$ and $\mathrm{Zn}(3 \mathrm{~d})$ orbits, respectively. In addition, the $\mathrm{Zn}(4 \mathrm{~s})-\mathrm{S}(3 \mathrm{p})$ hybridization at $4.3 \mathrm{eV}$ between the FNN Zn-S is observed.

Compared with pure $\mathrm{ZnS}$, the total DOS and partial DOS of Ag-doped ZnS in Fig.3[2] show that after importing $\mathrm{Ag}$ at the side of top of valence band there are redundant carrier-cavity pairs that induced an acceptor band near the Fermi level.

Table 1. Mulliken population of pure $\mathrm{ZnS}$ system

\begin{tabular}{ccccccccc}
\hline Species & $\mathrm{s}$ & $\mathrm{p}$ & $\mathrm{d}$ & $\mathrm{f}$ & Total & Charge $(\mathrm{e})$ & Bond & $\mathrm{S}-\mathrm{Zn}$ \\
\hline $\mathrm{S}$ & 1.82 & 4.65 & 0.00 & 0.00 & 6.47 & -0.47 & population & 0.49 \\
\hline $\mathrm{Zn}$ & 0.56 & 0.99 & 9.98 & 0.00 & 11.53 & 0.47 & Bond length $(\AA)$ & 2.360 \\
\hline
\end{tabular}

Table 2. Mulliken population of $\mathrm{Zn}$ And S of Ag-doped $\mathrm{ZnS}$ ( atomic population )

\begin{tabular}{cccccccc}
\hline Species & number & $\mathrm{s}$ & $\mathrm{p}$ & $\mathrm{d}$ & $\mathrm{f}$ & Total & charge (e) \\
\hline $\mathrm{S}$ & (1、4、6、7) I & 1.82 & 4.64 & 0.00 & 0.00 & 6.46 & -0.46 \\
& $(2 、 3 、 5 、 8) \mathrm{II}$ & 1.83 & 4.60 & 0.00 & 0.00 & 6.43 & -0.43 \\
\hline $\mathrm{Ag}$ & $\mathrm{I}$ & 0.66 & 0.59 & 9.78 & 0.00 & 11.02 & -0.02 \\
\hline \multirow{2}{*}{$\mathrm{Zn}$} & (1) I & 0.55 & 0.96 & 9.98 & 0.00 & 11.49 & 0.51 \\
& (2) II & 0.56 & 0.98 & 998 & 0.00 & 11.51 & 0.49 \\
& (5)III & 0.51 & 1.00 & 9.97 & 0.00 & 11.48 & 0.52 \\
& (3、4、6、7)IV & 0.52 & 0.99 & 9.97 & 0.00 & 11.48 & 0.52 \\
\hline
\end{tabular}

Table 3. Mulliken population of Ag-doped $\mathrm{ZnS}$ ( bond population )

\begin{tabular}{ccccccc}
\hline Bond & S I -Zn I & S I -Zn II & S I -Zn[V & S II -Zn III & S II -ZnIV & S II -Ag \\
\hline population & 0.48 & 0.50 & 0.44 & 0.47 & 0.51 & 0.37 \\
\hline Bond length $(\AA ̊)$ & 2.3654 & 2.3451 & 2.3676 & 2.3583 & 2.3435 & 2.4972 \\
\hline
\end{tabular}

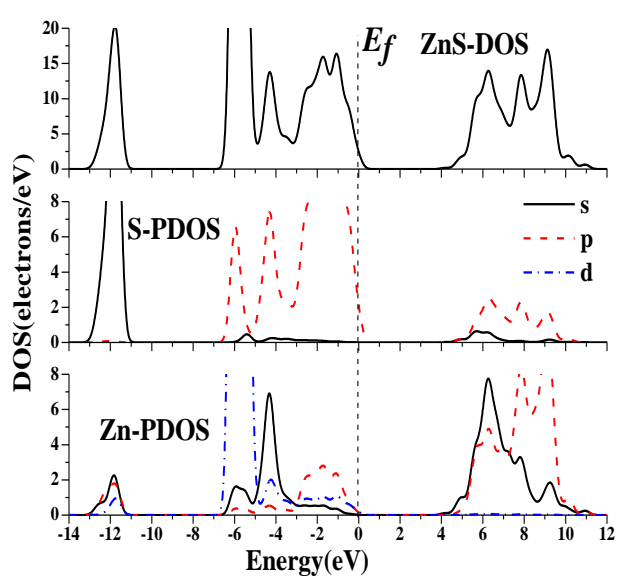

[1] $\mathrm{ZnS}$

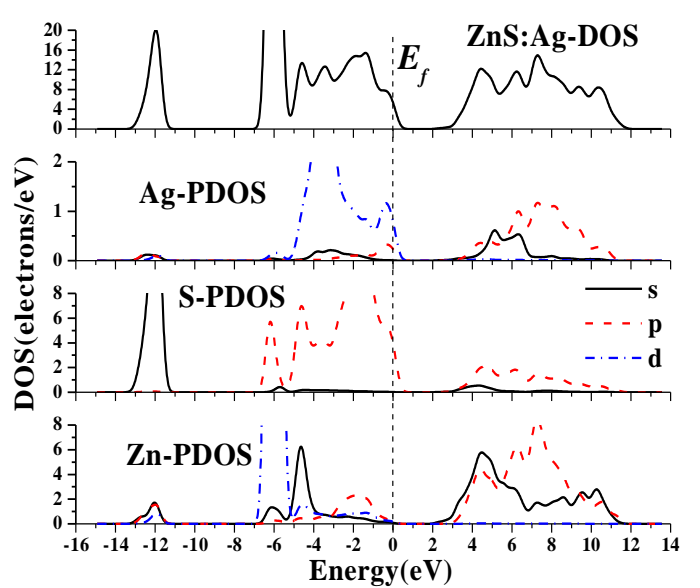

[2] Ag-doped ZnS

Fig.3 Calculated total and partial DOS of $\mathrm{ZnS}$ and Ag-doped $\mathrm{ZnS}$

There are high activity 4d state electrons in transition metal Ag. As shown in Fig.3, the density of 
states (DOS) for $\mathrm{Zn}$ in the pristine $\mathrm{ZnS}$ are almost the same as Ag-doped $\mathrm{ZnS}$, whereas, the DOS for $\mathrm{S}$, especially the partial density of states (PDOS) for S 3p state electrons, show significant changes. It is the doped $\mathrm{Ag}$ impurity nearest to those $\mathrm{S}$ atoms that shows a strong influence on the $\mathrm{S}$ atoms. Apparently, the DOS for doped systems illustrate obvious changes compared with pristine $\mathrm{ZnS}$ in the valence band (VB) top range. The impurity levels found on the VB top are mainly originated from the Ag-4d states hybridized with the S-3p states. These strong hybridization energy levels compose weakened localized type or dispersion type DOS which suggests high mobility of photogenerated carrier in the doped systems, which is mainly attributed to the contribution of $4 \mathrm{~d}$ state electrons of transition metal Ag.

The overlapped population is used to estimate that the bond is covalent or ionic. The bond with high overlapped population is covalent bond and the bond with low overlapped population has strong ionicity. Moreover the bigger population is, the stronger valence of bond is. The results in Table 2-3 show that the system of Ag-doped $\mathrm{ZnS}$ included not only covalent bonding but also ionic bonding. The covalency of S II -Ag bonds is weakest and population is smallest which made electrons of S close to $\mathrm{Zn}$. So population of S II $-\mathrm{ZnIV}$ is biggest, its covalency is strongest and its bond lengths are shortest. The bond lengths have low different values in the sort of S I - Zn I bond. This was because relative location of atoms made a tiny shift after importing impurity. But it did not influence a lot the analysis of state density.

\section{Conclusions}

The band structures, electronic structures and Mulliken populations of $\mathrm{ZnS}$ and Ag-doped $\mathrm{ZnS}$ have been computed by means of plane wave ultra-soft pseudopotential method with generalized gradient approximation which is based on the density functional theory. The thermal band gap of $\mathrm{ZnS}$ becomes small after Ag-doped. In the analysis of electronic structures, an acceptor band is induced because of doping Ag. It is pointed out that the acceptor level is hybridization due to the overlapping of the Ag-4d and S-3p. By analysis of the Mulliken populations, the systems of $\mathrm{ZnS}$ and Ag-doped $\mathrm{ZnS}$ include not only covalent bonding but also ionic bonding. After doping Ag, Zn-S bonds have strong covalence and the covalence of bond Ag-S is weakest after Ag-doped since its bond length is longest.

\section{Acknowledgements}

This work was financially supported by the National Natural Science Foundation of China (21373123).

\section{References}

[1] M. Bredal, J. Merikhi: J. Mat. Sci. Vol. 33 (1998), P.471-476.

[2] D. Jassby, M.Wiesner: Langmuir: the ACS journal of surfaces and colloids. Vol.27(2011), P.902-908.

[3] S. Kim, T. Kim, M. Kang, S.K. Kwak, T.W. Yoo, L.S. Park, I. Yang, S. Hwang, J.E. Lee, S.K. Kim et al: Journal of the American Chemical Society. Vol.134(2012), P.3804-3809.

[4] P. Jiang, J. Jie, Y. Yu, Z. Wang, C. Xie, X. Zhang, C. Wu, L. Wang, Z. Zhu, L. Luo: Journal of Materials Chemistry. Vol.22 (2012), P.6856-6861.

[5] C. Liu, Z. Liu, J. Li, Y. Li, J. Han, Y. Wang, Z. Liu, J. Ya: Microelectronic Engineering. Vol.103 (2013), P.12-16.

[6] I. R. Pala, S.L. Brock: ACS applied materials \& interfaces, Vol.4 (2012), P.2160-2167.

[7]Y. Liu, J. Hu, T. Zhou, R. Che, J. Li: Journal of Materials Chemistry. Vol.21(2011), P.16621-16627.

[8] H. Y. Ni, X.F.Cao, G.Z. Hu: Crystal Growth \& Design. Vol.7 (2007), P.280-285

[9] M. D. Segall, P. J. D.Lindan, M. J.Probert, C. J.Pickard, P. J. Hasnip, S. J.Clark, M. C. Payne: J. 
Phys. Condens. Matte. Vol.14 (2002), P.2717-2774.

[10] J. P. Perdew, K.Burke, M. Ernzerhof: Phys. Rev. Lett. Vol.77 (1996), P.3865-3868.

[11] T.H. Fischer, J. Almőf: J. Phys. Chem.Vol.96 (1992), P.9768-9774.

[12] H. J.Monkhorst, J. D. Pack: Phys. Rev. B Vol.13(1976), P.5188-5192.

[13] D. Gallagher, X. Hong, A. Nurmikko: Physical Rev. Lett., Vol.72 (1994), P. 416-419.

[14] R. M. Martin: Electronic Structure: Basic Theory and Practical Methods; (Cambridge University Press, Cambridge England 2004) 